\title{
Controlling der Marketingkommunikation: Zentrale Kennzahlen und ausgewählte Evaluationsverfahren
}

\author{
Zentrale Kennzahlen und ausgewählte \\ Evaluationsverfahren
}

\author{
Sven Reinecke, Simone Janz und Robert Hohenauer
}

\section{Inhalt}

1 Erfolgsnachweis und Steuerung der Marketingkommunikation $\ldots \ldots \ldots \ldots \ldots \ldots \ldots \ldots \ldots$

2 Kommunikationskontrollen und -audits als Formen der

Kommunikationsüberwachung .......................................... 6

3 Zentrale Kennzahlen und ausgewählte Evaluationsverfahren der

Kommunikationskontrolle ........................................... 10

4 Fazit: Wirksamkeit und Wirtschaftlichkeit der Kommunikation durch Controlling

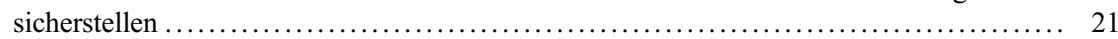

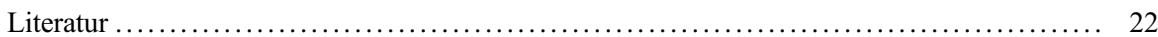

\section{Zusammenfassung}

Ein umfassendes Controlling hilft, die Wirksamkeit und die Wirtschaftlichkeit von Marketingkommunikationsmaßnahmen zu optimieren. Ohne systematische Erfolgssteuerung bleiben Fehlentwicklungen unerkannt. Der nachfolgende Artikel gibt einen Überblick über die wichtigsten Methoden, Kennzahlen und Evaluationsverfahren.

\section{Schlüsselwörter \\ Marketingcontrolling • Kennzahlen • Performance Measurement • Evaluation • Kommunikation}

S. Reinecke $(\square) \cdot$ R. Hohenauer

Institut für Marketing, Universität St. Gallen, St. Gallen, Schweiz

E-Mail: sven.reinecke@unisg.ch; robert.hohenauer@unisg.ch

S. Janz

Strategische Unternehmensplanung/Strategic Corporate Planning, Audi AG, Ingolstadt, Deutschland

E-Mail: simone.janz@audi.de 


\section{$1 \quad$ Erfolgsnachweis und Steuerung der Marketingkommunikation}

\subsection{Die Notwendigkeit, den Wertbeitrag des Marketings zu messen}

Den Wertbeitrag der Marketingkommunikation sowie dessen Effektivität und Effizienz nachzuweisen - dies ist aufgrund zahlreicher Störfaktoren und oft unklarer UrsacheWirkungszusammenhänge eine zentrale Herausforderung: Ohne systematische Erfolgssteuerung und -kontrolle bleiben Einsparpotentiale und Fehlentwicklungen unerkannt. Ferner hat der Legitimationsdruck auf Marketing- respektive Kommunikationsmanager, deren Budgets zurzeit häufig noch als ,Kosten“ und damit als potentielle Streichposten gelten (Doyle 2000, S. 307 ff.), in den letzten Jahren stark zugenommen (Reinecke 2014, S. 11). Professionelles Controlling erhöht die Wirtschaftlichkeit des Marketing und verbessert die Integration des Marketing im Unternehmen. Darüber hinaus generiert ein umfassendes Controlling eine sichere Argumentationsgrundlage, wenn es um die Verteilung von Budgets geht (Huhn und Sass 2011, S. 7).

Die Optimierung zentraler Unternehmensziele wie Gewinn, Sicherheit, Wachstum oder Shareholder Value erfordert eine konsequente Sicherstellung der Effektivität und Effizienz sämtlicher Organisationseinheiten und -prozesse. Nicht umsonst hat Controlling - nach Weber und Schäffer (2008) verstanden als Sicherstellung der Rationalität bzw. der Effektivität (Wirksamkeit) und Effizienz (Wirtschaftlichkeit) der Unternehmensführung - in den vergangenen Jahren einen starken Bedeutungszuwachs erfahren. Immer mehr Unternehmensbereiche müssen sich heute damit auseinandersetzen, welchen Beitrag sie zur Erreichung der Unternehmensziele leisten bzw. welchen Wert sie für das Unternehmen generieren (u. a. Day und Fahey 1988, S. 45). Dies gilt zunehmend auch für einen Bereich, der lange von dieser Forderung verschont geblieben ist: das Marketing (u. a. Rust et al. 2004; O'Sullivan und Abela 2007, S. 79) und insbesondere die Marketingkommunikation (Vidale und Wolfe 1957; Blair und Schroiff 2001, S. 52 ff.), auf die in vielen Unternehmen ein Großteil des Marketingbudgets entfällt. Somit ist „Wertschöpfung durch Kommunikation (...) eines der zentralen Themen der Kommunikation im 21. Jahrhundert" (Huhn und Sass 2011, S. 6 ff.). Die grösste Herausforderung im Marketing ist dessen Umsetzung (Backhaus und Voeth 2009, S. 31), vor allem hinsichtlich des Controllings.

\subsection{Controlling, Performance Measurement und Management}

Ein systematisches Controlling der Marketingkommunikation kann sicherstellen, dass Entscheidungen unter geringerer Unsicherheit getroffen werden, diese und ein entsprechender Budgeteinsatz besser legitimiert und mittel- bis langfristig der Wertbeitrag der Marketingkommunikation aufgezeigt werden kann. Dies erfordert jedoch auch die Bereitschaft des Top-Managements und des zentralen Controllings, im 
Sinne der Leistungstransparenz dort mit kommunikationsrelevanten Kennzahlen zu arbeiten, wo keine Ursache-Wirkungszusammenhänge zwischen Kommunikationskennzahlen und monetären Kennzahlen nachgewiesen werden können.

Grundsätzlich können für das Controlling der Marketingkommunikation die unternehmensweit bereits vorhandenen Controllingansätze und -instrumente auf den Kommunikationsbereich übertragen bzw. an diesen angepasst werden. Beispiele für eine konsequente Übertragung gibt es in der Literatur allerdings wenige. Der Themenbereich wird jedoch unter dem Begriff ,Kommunikationscontrolling' oder ,Werbecontrolling' thematisiert, wobei unter dem Begriff, Kommunikationscontrolling' entwickelte Modelle in der Regel dem Controlling der an alle Stakeholder gerichteten Kommunikation (u. a. Corporate Communication) dienen (Übersicht z. B. bei Huhn und Sass 2011, S. 8). Sowohl im Bereich der Marketingkommunikation als auch im Bereich der an alle Stakeholder gerichteten Kommunikation ist jedoch eine Entwicklung weg vom Begriff ,Controlling' hin zum ,Performance Measurement ${ }^{\star}$ und ,Performance Management ${ }^{\star}$ zu erkennen, da Aspekte wie Anschlussfähigkeit an unternehmensweite Systeme (wie z. B. die Balanced Scorecard), hierarchieübergreifende Zielkaskade und Strategieimplementierung im Fokus stehen müssen, um eine strategie- und zielorientierte Steuerung sicherstellen zu können (z. B. Reinecke 2004; Brettschneider et al. 2007; Janz 2009). Performance Measurement wird nachfolgend als ein Teilbereich des Controllings verstanden, der sich primär mit Kennzahlen auseinandersetzt (für das Marketing siehe Farris et al. 2010). Performance Management geht dagegen zum Teil über Controllingaspekte hinaus, weil es auch Managemententscheidungen integriert (ausführlich Reinecke 2004).

Controlling wird von Managern und Controllern wahrgenommen und dient der Reduktion der Entscheidungsunsicherheit. Ziel ist es, die Effektivität (Wirksamkeit) und Effizienz (Wirtschaftlichkeit) des Managements sicherzustellen (ausführlich z. B. Weber und Schäffer 2008; Reinecke und Janz 2007; Lasslop 2003). Während sich die Effektivität auf die erzielten Outputs relativ zu der Erreichung a priori festgelegter Ziele bezieht, fokussiert die Effizienz die Relation zwischen Output und Input. Eine Maßnahme ist effizient, wenn es zu einem Output/Input-Verhältnis einer Maßnahme keine andere Maßnahme gibt, die ein besseres Verhältnis erzielt, wobei der Output den Input übersteigen muss.

Aufgaben, die das Marketingcontrolling und somit auch der Teilbereich ,Controlling der Marketingkommunikation' zur Erfüllung dieser Rolle theoretisch wahrnimmt, lassen sich in vier Kategorien zusammenfassen: problembezogene Informationsversorgung (v. a. Informationen aus Marktforschung und internem Rechnungswesen), Unterstützung der strategischen und operativen Planung, Marketingüberwachung (Kontrollen und Audits) sowie führungsübergreifende Koordination (z. B. Erstellen von Kennzahlensystemen). In Unternehmen liegt der Schwerpunkt des Controllings der Marketingkommunikation erfahrungsgemäß auf der Kontrolle und Messung des Kommunikationserfolgs (siehe nachfolgend) und damit einhergehend - über einen Feedbackprozess in einem iterativen, zyklischen Planungsregelkreis - auf der Planungsunterstützung: So ist das Ziel der Überwachung (Kontrollen und Audits) die Optimierung der Planung und Realisierung. In diesem Rahmen erfolgt in der Regel auch die problembezogene Informationsversorgung. 


\section{Kommunikationskontrollen und -audits als Formen der Kommunikationsüberwachung}

Marketingkontrollen sind ein zentrales Aufgabengebiet von Marketingcontrolling und Marketingmanagement. Als solches erfüllen sie zwei Rollen: Zum einen sind sie unverzichtbarer Bestandteil der Planung, zum anderen deren Gegenpart. Kontrolle und Planung sind ferner sowohl Voraussetzungen als auch Instrumente der Koordination (Horváth 2012).

Ergebniskontrollen sind in erster Linie Abweichungsanalysen und liefern Informationen, inwieweit (Wirkungskontrollen), wie effizient (Wirtschaftlichkeitskontrollen) und mit welchem Grad der Budgeteinhaltung (Kosten- und Budgetkontrollen) die Ziele mit den verfolgten Strategien (v. a. den eingesetzten Maßnahmen) erreicht wurden (siehe auch Hermanns und Marwitz 2008). Diesen schließt sich in der Regel eine Analyse von Abweichungsursachen und das Auslösen von Maßnahmen zur Korrektur von Planabweichung oder falls erforderlich das Anpassen von Planwerten an. Eine Kontrolle, die allein auf einen Vergleich von Soll- und Ist-Werten abzielt, greift für die Sicherstellung der Führungsrationalität jedoch zu kurz: Eine zielgerichtete Durchsicht der Planungsgrundlagen und der Planungssystematik unterbleibt. Somit sind Soll-Ist-Vergleiche nur im Rahmen einer ganzheitlichen ,Überwachung ‘ ein sinnvoller Kontrollmechanismus. Nur dann wird sichergestellt, dass der strategische Plan insgesamt sinnvoll ist; Ergebniskontrollen müssen deshalb durch Audits ergänzt werden (u. a. Böcker 1988, S. 49). In Anlehnung an Kotler und Keller (2012, S. 643) kann ein Marketingaudit als eine umfassende, systematische, nicht weisungsgebundene, regelmässige Untersuchung von Marketingumwelt, -zielen, -strategien sowie von Marketingprozessen, -organisation und -massnahmen einer strategischen Geschäftseinheit definiert werden. Diese setzen im Vergleich zu Ergebniskontrollen inhaltlich auf einer Metaebene an, da nicht die Abläufe und Ergebnisse an sich, sondern das Marketing- und Kommunikationssystem und dessen Rahmenbedingungen Objekt der Überwachung sind (ausführlich u. a. Böcker 1988; Köhler 2006). Ferner sollte mit dem Ziel der Früherkennung eine permanente strategische Überwachung erfolgen.

Die Systematik einer umfassenden Überwachung im Marketing weist somit folgende Elemente auf (ausführlich u. a. Reinecke und Janz 2007, S. 140 ff.): Ergebniskontrollen als konkrete Soll-Ist-Vergleiche (Effektivitäts-, Effizienz- sowie Kosten- und Budgetkontrollen), Ablaufkontrollen, Marketingaudits und die strategische Überwachung (als offener, ungerichteter Beobachtungsradar).

Analog können Kontrollen und Audits der Marketingkommunikation wie in Abb. 1 dargestellt systematisiert werden, wobei das Kommunikations-Benchmarking neben den zuvor genannten Instrumenten immer wichtiger wird (ausführlich Reinecke und Janz 2007, S. 269). Audits werden zugunsten einer ausführlichen Diskussion der Effektivitätskontrolle im Folgenden nicht berücksichtigt; sie können jedoch analog zu generellen Marketingaudits erfolgen (Reinecke und Janz 2007, S. 146 ff.).

Um die Grundlagen umfassend darzustellen, wird nachfolgend vor allem auf die Werbung als Kommunikationsinstrument Bezug genommen. Grundsätzlich lassen 


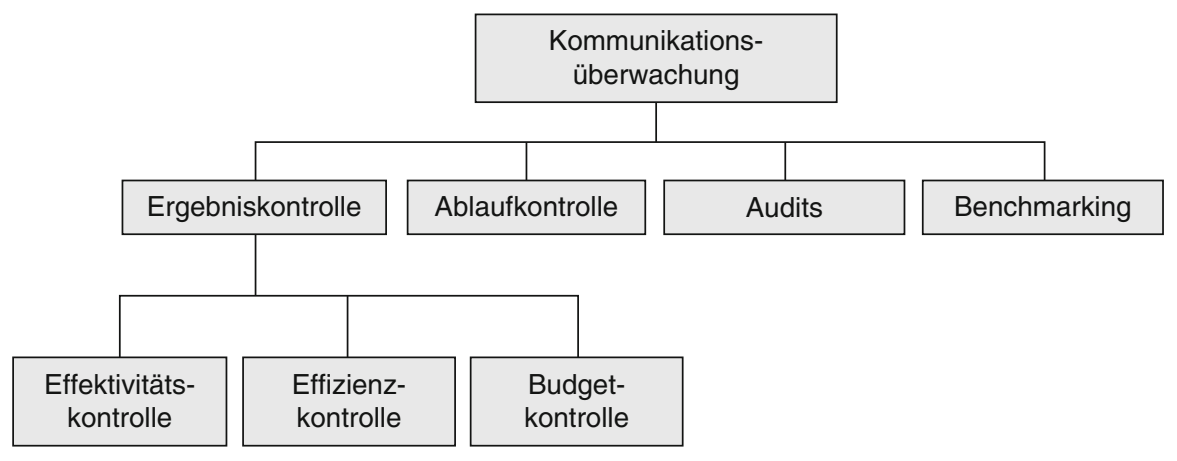

Abb. 1 Formen der Kommunikationsüberwachung (Quelle: Reinecke und Janz 2007, S. 221)

sich die Ausführungen jedoch auch auf Kontrollen und Audits anderer Kommunikationsinstrumente wie Public Relations, Sponsoring oder die Online-Kommunikation übertragen. Die Online-Kommunikation (z. B. Suchmaschinenmarketing, Bannerwerbung, Social Media- und Internetpräsenz) wird aufgrund nachfolgend bewusst ausgeklammert, da sich hierfür spezifische Controllinginstrumente entwickelt haben (vgl. Esch und Eichenauer Verfahren zur Messung der Kommunikationswirkung im Internet und bei Social Media).

Ziel von Effektivitätskontrollen ist es, die monetären und nichtmonetären Kommunikationswirkungen durch den Vergleich zwischen geplanten und realisierten Werten zu ermitteln. Entsprechende Verfahren der Kommunikationskontrolle können grob nach folgenden Kriterien geordnet werden:

- der Wirkungsstufe, auf der sie ansetzen (z. B. Messung der Wahrnehmung oder der Einstellung, Abb. 2),

- dem verwendeten Messverfahren (z. B. Beobachtung oder Befragung, Abb. 2)

- oder einer bestimmten Art des Messzeitpunkts (z. B. Pretest oder Posttest bzw. Tracking, Tab. 1).

Die Darstellung in Abb. 2 entspricht einer praktischen Denkweise: Welche Kenngröße lässt sich wie messen, und gibt es evtl. andere Kennzahlen oder Messverfahren?

Mögliche Untersuchungsdesigns greifen auf die genannten Kennzahlen und entsprechenden Messverfahren zurück. Sie können ferner mittels der in Tab. 1 genannten Kriterien gestaltet und kombiniert werden (ausführlich z. B. Schwaiger und Trommsdorf zu Pretest- und Tracking-Angeboten von Marktforschungsunternehmen; Pretests: Trommsdorff 2003, S. 144 ff.; Schwaiger 2006, S. 543 f.; Trackings und damit implizit Posttests: Schwaiger 2006, S. 547 f.).

Demgegenüber wird mittels Effizienzkontrollen der Erfolg einer Kommunikationsmaßnahme unter wirtschaftlichen Nutzen-Kosten- bzw. Output-Input-Aspekten beurteilt. Auch kann die Vorteilhaftigkeit einer Kommunikationsmaßnahme verglichen mit anderen Optionen festgestellt werden. Hierzu eignen sich insbesondere Kennzahlen, die quantitativ erfassbare Sachverhalte in konzentrierter Form relevant 


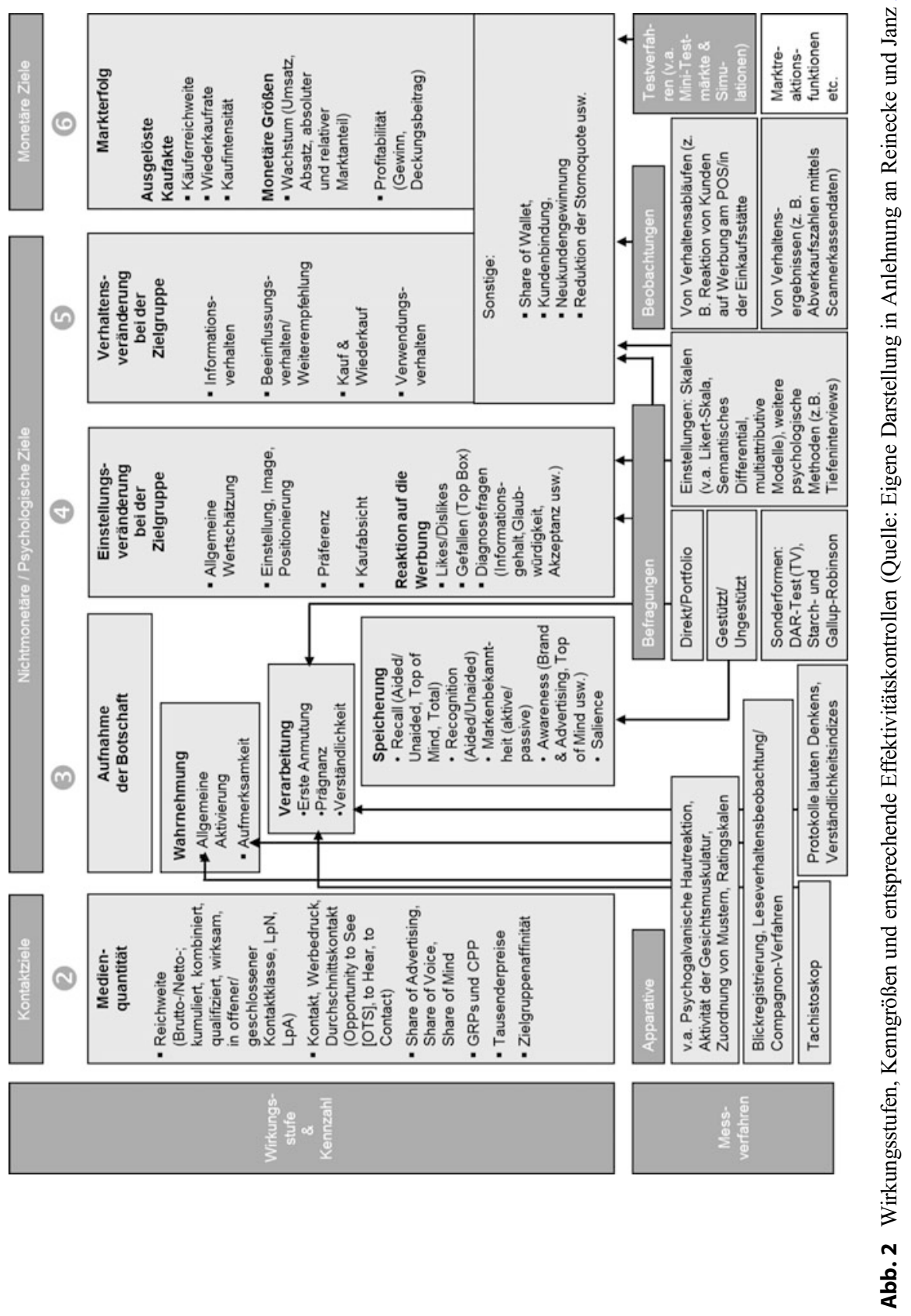


Tab. 1 Mögliche Untersuchungsdesigns von Effektivitätskontrollen: Gestaltungs-kriterien und mögliche Ausprägungen

\begin{tabular}{l|l}
\hline Kriterium & Ausprägung \\
\hline Zeitpunkt der Messung & Pretests (vor Durchführung) \\
& $\begin{array}{l}\text { Posttests (nach Durchführung) } \\
\text { Trackings (kontinuierlich) }\end{array}$ \\
\hline Gegenstand & Kommunikations-Mix \\
& Gesamte Kampagne (Kampagnentest) \\
& Einzelne Massnahmen (Sujet-Test, Motiv-Test) \\
\hline Ort & Laborbedingungen \\
& Im Feld \\
\hline Art der Testperson-Auswahl & Recruited \\
& Self-selected \\
\hline Air-Phase & On-Air \\
& Off-Air \\
\hline Anzahl der Werbemittelkontakte & Einmalig \\
& Mehrmalig \\
\hline Programmeinbettung & In-Program (Test-Werbemittel eingebettet in Programm/ \\
& Inhalt oder weitere Werbemittel) \\
& Naked \\
\hline Aufbau & Nur Posttest \\
& Pretest (Nullmessung) und Posttest \\
\hline
\end{tabular}

(Quelle: Eigene Darstellung)

und knapp festhalten (Siegwart et al. 2010). Analog zu den Kommunikationswirkungsstufen lassen sich grundsätzlich drei Ansatzpunkte für die Entwicklung eines Kennzahlensystems (weitergehend Bauer et al. 2000b, S. 15 ff.) bzw. drei Werbekennzahlenebenen unterscheiden (Behrens 1996, S. 147, S. 275; Bauer et al. 2000a, S. 5; 2000b, S. 15 ff.):

- Kostenbezogene Kennzahlen: Kontaktgrößen, psychologische Größen und/oder Ertragsgrößen in Relation zu den eingesetzten Kommunikationskosten (z. B. Mediaspendings).

- Kontaktgrößenbezogene Kennzahlen: psychologische und/oder Ertragsgrößen im Verhältnis zu erzielten Kommunikationskontakten in der Zielgruppe.

- Kennzahlen bezogen auf die psychologische Wirkung: Ertragsgrößen oder einzelne psychologische Größen in Relation zu (anderen) zielrelevanten psychologischen Wirkungsgrößen (z. B. Kaufbereitschaft (\%)/Bekanntheitsgrad (\%)).

Die Kosten- und Budgetkontrolle ist neben der allgemeinen Ergebniskontrolle die in der Praxis am stärksten verbreitete Kontrollform und gleichzeitig das mit Abstand am häufigsten eingesetzte Instrument des Marketingcontrollings (Reinecke 2014, S. 28); dies unter anderem, weil sie - zumindest bei formal und nicht inhaltlich ausgerichteten Kontrollverfahren - verhältnismäßig unaufwändig durchgeführt werden kann. Gegenstand der Kostenkontrolle sind die Kosten pro Mengeneinheit. Kumulierte Kosten pro Zeiteinheit sind Gegenstand von Budgetkontrollen. Im 
Rahmen einer formalen Budgetkontrolle werden lediglich die Planbudgets an dem Grad ihrer Einhaltung gemessen. Aussagen über die Zweckmäßigkeit oder die sachliche Verwendung der Budgethöhe sind nicht möglich. Bei der inhaltlichen Kontrolle werden dem Budget auch marketingrelevante Erfolgs- und Effizienzgrößen gegenübergestellt (u. a. Böcker 1988, S. 155 f.; nicht in jedem Unternehmen existiert neben einem Marketingbudget ein Kommunikationsbudget). Dies ist meist mit Abweichungs- (Preis- oder Mengenabweichungen) und Wertanalysen (z. B. mittels Gemeinkostenwertanalyse) verbunden, wobei Letztere der Bestimmung der Angemessenheit der Höhe des Marketingbudgets und der Aufdeckung von Kostensenkungspotenzialen dienen.

Kommunikations-Ablaufkontrollen umfassen die Fortschritts- und Terminüberwachung bei der Entwicklung und Durchführung einer Kommunikationskampagne (Bauer et al. 2000b, S. 28). Diese Aufgaben sind im Kommunikationsbereich häufig aufwändiger und komplexer als in anderen Bereichen, weil zahlreiche Schnittstellen zu koordinieren sind: zum Beispiel Schnittstellen zwischen Auftraggeber- und Agenturseite sowie zwischen Auftraggeber, Agentur und weiteren Organisationen wie Media-Agenturen oder Produktionsstudios.

\section{Zentrale Kennzahlen und ausgewählte Evaluationsverfahren der Kommunikationskontrolle}

\subsection{Systematisierung grundlegender Wirkungsmodelle und Zielsysteme anhand des AIDA-Schemas}

Um die zentralen Ziel- und damit Kontrollgrößen der Kommunikationskontrolle sowie entsprechende Messverfahren strukturiert vorzustellen, wird den nachfolgenden Ausführungen Abb. 2 zugrunde gelegt, welche nach dem AIDA-Schema (Attention-Interest-Desire-Action) organisiert ist. Dieses wurde zwar zu recht kritisiert (siehe Reinecke und Janz 2007, S. 227 ff. zu Kritik und Weiterentwicklung; ausführlich auch Kroeber-Riel und Gröppel-Klein 2013, S. 676 f.), es enthält jedoch wesentliche Dimensionen und eignet sich deshalb für Systematisierungszwecke.

Definitionsgemäß richten sich die Ziele der Kommunikation stets auf die Beeinflussung des Verhaltens auf dem Markt. Auch in der Praxis wird der Nachweis des Beitrags der Kommunikation zu der Erreichung von unternehmenserfolgsrelevanten Zielgrößen wie Wachstum (Umsatz [Preis x Absatzmenge], absoluter und relativer Marktanteil) und Profitabilität (Gewinn, Deckungsbeitrag) gefordert, welche durch das Marktverhalten beeinflusst werden. Insbesondere im Kommunikationsbereich ist jedoch die Unterscheidung in nichtmonetäre, vor allem psychologische, und monetäre Zielgrößen zentral. Dies wird in den genannten Wirkungsmodellen abgebildet:

Ziele des Marketings, die mittels Kommunikation unterstützt werden sollen, lassen sich häufig nicht direkt realisieren, beispielsweise die Einführung eines neuen Produkts, die Erhaltung des Kundenstamms, die Umsatz- bzw. Preisniveaustabilisierung oder der Ausbau von Marktanteilen. Vielmehr ist zur Erreichung dieser Ziele der Einsatz diverser Einzelmaßnahmen notwendig, die zieladäquat ausgewählt 
werden müssen. Für diese müssen instrumentenspezifische Ziele formuliert werden. So müssen zur Erhöhung des Umsatzes, insofern dies überhaupt rein kommunikativ möglich ist, verschiedene Kommunikationstechniken (und -instrumente) eingesetzt und kombiniert werden: Beispielsweise die emotionale Konditionierung eines Markennamens durch einen TV-Spot, die Veränderung der Einstellungen (insbesondere von Konsumenten) durch die Werbebotschaft oder die Verstärkung vorhandener Verhaltensweisen durch einen einprägsamen Slogan. Nur wenn konkrete Kommunikationsziele als Subziele formuliert werden, kann Kommunikation geplant durchgeführt und ihre Wirkung anhand des Zielerreichungsgrades kontrolliert werden (Kroeber-Riel und Gröppel-Klein 2013, S. 675 f.).

Dies hängt damit zusammen, dass sich zwischen der Kommunikation als Input und dem Kaufverhalten als Output eine Reihe psychologischer Vorgänge abspielt, denen zum einen eine Prädikatorfunktion für das nachfolgende Kaufverhalten zukommt. Zum anderen ist der direkte Nachweis der Wirkung einer Kommunikationsmaßnahme auf das Verhalten aufgrund zahlreicher nichtkontrollierbarer Einflussfaktoren grundsätzlich schwierig. Gleichzeitig werden nichtmonetäre Zielgrößen wesentlich weniger von anderen Unternehmens- oder Konkurrenzmaßnahmen sowie weiteren Umweltfaktoren beeinflusst, so dass hier eine direktere Messung und Ursache-Wirkungs-Zuordnung möglich ist.

Mittlerweile wird davon ausgegangen, dass die Richtung der Wirkung zwischen den verschiedenen Stufen des Modells nicht zwangsläufig einseitig (in Abb. 2 von links nach rechts) ist, sondern auch rückwärts gerichtet sein kann. So wirkt sich beispielsweise das Kaufverhalten häufig auch auf die Einstellung aus, beispielsweise infolge der Nutzung der erworbenen Leistung. Die Wirkungen auf den einzelnen Stufen werden von weiteren Faktoren beeinflusst, die situationsspezifisch berücksichtigt werden müssen: Grad des Involvements (High, Low), Art der Kommunikation (informativ, emotional oder gemischt; siehe Kroeber-Riel und Esch 2011), Persönlichkeitsmerkmale der Rezipienten (Temperament, Werbeaffinität, Empfänglichkeit und Einstellung gegenüber Werbung, Gemütszustand, Werbekontext, bereits vorhandene Kauf- und Nutzungserfahrungen, Zeiteffekte, kulturelle Gegebenheiten) und ganz allgemein die situativen Gegebenheiten der Umwelt (u. a. Kroeber-Riel und Gröppel-Klein 2013, S. 677). In konkreten Testsituationen wird versucht, diese Größen weitgehend zu berücksichtigen und wenn möglich zu kontrollieren. Die einzelnen Messverfahren werden im Folgenden nicht ausführlich dargestellt. Der Fokus liegt vielmehr auf einer logischen und managementorientierten Darstellung der zentralen Zusammenhänge.

\subsection{Kommunikationsaufwand, Kommunikationsbudget und Kommunikationsqualität als unabhängige Größen der Werbewirkungsmessung}

Der Kommunikationsaufwand bzw. das Kommunikationsbudget sowie die Kommunikationsqualität (v. a. Kommunikationsinhalt, - botschaft und -gestaltung sowie Innovation und Medienqualität) stellen in diesem Modell die unabhängigen (Input) Variablen dar. Die Qualität der Werbung im Hinblick auf kreative Idee und 
Umsetzung ist eine Größe, die in der Diskussion um Werbewirkungen teilweise außer Acht gelassen wird, obwohl es effizienter ist, die Zielgruppe seltener, jedoch mit effektiverer bzw. qualitativ besserer Werbung zu erreichen (Ambler 2000; Cramphorn 2004, S. 171). Angesichts einer derartigen Steuerungslücke können seit wenigen Jahren Bemühungen großer Werbetreibender beobachtet werden, auch den Beitrag der Kreativität zum Kommunikationserfolg im Rahmen der Kommunikationsplanung zu berücksichtigen: Der Konsumgüterhersteller Procter \& Gamble hat 2003 beispielsweise entsprechende Kenngrößen (z. B. watchability scores) in seine Copy-Test-Protokolle aufgenommen (Neff 2005).

Als Voraussetzung von Kommunikationswirkungen ist zunächst der Kontakt der Zielgruppe mit dem Werbemittel in den Medien oder zum Beispiel dem Event erforderlich, wobei in Massenmedien ein Mindest-Werbedruck (mindestens konkurrenzfähig) erforderlich ist. Kontaktplanung erfolgt in Media-Agenturen oder -Abteilungen auf Basis der Mediadaten der einzelnen Werbeträger. Zur Generierung und Evaluation alternativer Mediapläne werden in der Regel Verfahren wie Modelling (ökonometrische Modelle, die Prognosen mittels Vergangenheits- und Schätzwerten erlauben), Evaluierungsmodelle und Rangreihen eingesetzt (ausführlich: der Beitrag zu ,Mediaplanung' in diesem Buch sowie Reinecke und Janz 2007, S. 240 ff.).

\subsection{Awareness, Recall, Recognition und Bekanntheit als Zielgrößen der Werbewirkungsmessung}

Dem Kontakt folgt idealerweise die Aufnahme der Botschaft. Sie umfasst die Wahrnehmung, Verarbeitung und Speicherung der Botschaft durch die Zielpersonen als Voraussetzung dafür, dass Wirkungen von gespeicherten Informationen (wie die Beeinflussung von Emotionen und Einstellungen) auf der nächsten Stufe stattfinden können.

Dieser dritten Stufe liegt das Dreispeichermodell der Informationsverarbeitung zugrunde. Demnach erfolgt die gedankliche Verarbeitung von Reizen mittels folgender Gedächtnisstrukturen, die häufig als Speicher bezeichnet werden (ausführlich u. a. Behrens 1996, S. 294 ff.; Kroeber-Riel und Gröppel-Klein 2013, S. 307 ff.; Neumann 2013a, S. 58 ff.): (1) Sensorischer Informationsspeicher bzw. Ultrakurzzeitspeicher, (2) Kurzzeitspeicher und (3) Langzeitspeicher. Wesentlich ist, dass die Zielperson Kontakt mit einem Reiz haben muss, dieser nicht nur mit den Sinnesorganen erfasst (sensorischer Informationsspeicher) wird, sondern auch so viel Aufmerksamkeit erzeugen muss, dass er als Reiz wahrgenommen wird. Dadurch kann er in das Bewusstsein bzw. den Kurzzeitspeicher gelangen, wo er folgende erfolgsrelevante Wirkungen auslösen kann:

- Allgemeine Aktivierung: Diese ist notwendig, damit die Vorgänge in den anderen Speichern effizienter ablaufen.

- Informationsverarbeitung (emotional und kognitiv): Die ins Bewusstsein gelangenden Informationen werden unterschiedlich intensiv wahrgenommen (flüchtig bis klar und bewusst) und emotional bewertet. Ebenso werden sie kognitiv (gedanklich) verarbeitet bzw. in die vorhandenen Gedankenstrukturen eingeordnet. 
Nur wenige Informationen gelangen aus dem Kurzzeitspeicher in den Langzeitspeicher, viele gehen wieder verloren (u. a. Kuß und Tomczak 2007, S. 27 f.). Informationen im Langzeitspeicher sind gelernt bzw. langfristig gespeichert und messbar (Neumann 2013a, S. 58 f.; Kroeber-Riel und Gröppel-Klein 2013, S. 55 ff.)

- Wissen (Bekanntheit, Erinnerung/Recall, Wiedererkennen/Recognition),

- Gefuihle (Emotionen als ungerichteter Zustand und Einstellung als Emotion verbunden mit Zielorientierung in Bezug auf Verhalten und Gegenstandsbeurteilung) und/oder

- Verhaltensintentionen.

Durch eine gezielte Steuerung dieser Lernprozesse (mittels Vermittlung von Wissensinhalten oder unterschiedlichen Arten von Konditionierung) können vorhandene Einstellungen und Handlungsabsichten modifiziert oder neu generiert werden, da Einstellungen ein Ergebnis dieser Lernprozesse sind (z. B. Neumann 2013a, S. 58 f., S. 132 f.). Dementsprechend sind Gegenstände der nächsten Wirkungsstufe die Einstellungsänderung und die Änderung von Handlungsabsichten.

Bevor dies näher erläutert werden kann, soll zunächst die Recall- (Erinnern) und Recognitionmessung (Wiedererkennen) vertieft werden, da dies in der Praxis bis heute die bekannteste Methode ist, um Werbewirkungen zu ermitteln (u. a. Fenwick und Rice 1991, S. 23; Krishnan und Chakravarti 1999, S. 4 ff.). Um ein differenzierteres Bild zu ermöglichen, sollten jedoch stets weitere Wirkungen erhoben werden (u. a. Nickel 2001, S. 70).

Grundsätzlich ist Awareness die zentrale Kenngröße zur Evaluation des Wissens, welche weitergehend in Kenntnis des Werbemittels (Ad Awareness) und Kenntnis der Marke (Brand Awareness bzw. Markenbekanntheit) differenziert wird (z. B. Bergkvist 2000, S. 37 ff.). Beides wird in der Regel über das Erinnern (Recall) und das Wiedererkennen (Recognition) abgefragt (Ellinghaus 2000, S. 27 ff.). Allerdings vertreten einzelne Autoren die Auffassung, Recognition würde nur bei Werbemitteln abgefragt werden (z. B. Erichson und Maretzki 1993, S. 547); auch wird im Zusammenhang mit Marken und Produkten häufig nur der Recall erhoben. Grundsätzlich lässt sich jedoch auch die Recognition bei Produkten und Marken einsetzen (u. a. Bergkvist 2000, S. 37 ff.). Ferner wird bei den Begriffen Recall und Awareness zwischen Aided/Unaided (gestützt/ungestützt) sowie Top of Mind und Total unterschieden. Ein weiterer Begriff, der in diesem Zusammenhang gebräuchlich ist, ist die Salience. Diese Größe wird als Oberbegriff für Top of Mind-Awareness, Unaided Awareness und Total Awareness (Unaided plus Aided) verwendet (Haley und Baldinger 2000, S. 116). Mittels standardisierter Befragungen werden somit

- Unaided Recall (ungestützte Erinnerung an ein bestimmtes Produkt, eine Marke oder ein bestimmtes Werbemittel usw.),

- Aided Recall (gestützte Erinnerung an ein bestimmtes Produkt, eine Marke oder ein bestimmtes Werbemittel usw.; Werberecall = Advertising Awareness) und

- Recognition (Wiedererkennen eines bestimmten Produkts, einer Marke oder eines bestimmten Werbemittels usw.) erhoben. 
„Erinnern“ bedeutet hier, dass ausreichend Einzelheiten beschrieben werden können, um Verwechslungen ausschließen zu können. Die Unterscheidung zwischen Aided und Unaided Recall bezieht sich darauf, ob das Gelernte frei und ohne Unterstützung (Unaided; auch Unprompted oder Spontaneous genannt) oder mit Gedächtnisstützen (Aided, auch Prompted genannt), wie zum Beispiel Hinweise auf den Kontext des gelernten Materials, wiedergegeben werden kann. Die Marke, die bei einer Frage zur Überprüfung des Unaided Recall als erstes genannt wird (teils auch die beiden zuerst genannten Marken), ist Top of Mind, so dass in diesem Zusammenhang von Top of Mind Recall und Top of Mind Awareness gesprochen wird. Beim Wiedererkennen wird der befragten Person das gelernte Material (z. B. eine Werbeanzeige) zusammen mit anderem Material vorgelegt und sie wird gefragt, an welches Material sie sich erinnert. Bei Marken erfolgt dies, indem den Testpersonen Listen mit bis zu 30 Marken vorgelegt werden und diese angeben, welche Marke sie kennen bzw. von welcher sie schon einmal etwas gehört haben. Der Begriff „Total“ (Total Recall oder Total Awareness) drückt die Summe von Aided und Unaided Werten aus.

Im Allgemeinen sind gute Recall- und Recognition-Werte notwendige, nicht jedoch hinreichende Bedingungen für positive Werbewirkungen. Bei der Interpretation sind folgende Aspekte zu berücksichtigen:

- Die Werte müssen stets in Relation zu angemessenen Vergleichsgrößen gesehen werden, da sie zum Beispiel stark von dem Verwenderanteil der Produkte und der Positionierung abhängig sind (Kloss 2003, S. 163). Insbesondere sollten sie nur im Zusammenhang mit Einstellungswirkungen gemessen werden, da hohe Recall- und Recognitionwerte beispielsweise auch im Zusammenhang mit negativen Emotionen stehen können (wie im bekannten Fall der Marke Benetton) (Nieschlag et al. 2002, S. 1114).

- Ferner müssen Gedächtniswerte, die sich bei den unterschiedlichen Methoden ergeben, differenziert interpretiert werden (Neumann 2013b, S. 193 ff.), weil sie unterschiedliche gedankliche Abrufleistungen aus dem Gedächtnis erfordern (Kroeber-Riel und Gröppel-Klein 2013, S. 454): Der Wert des Unaided Recall ist am niedrigsten, der Recognition-Wert am höchsten, gleichzeitig korrelieren diese Werte stark (Stapel 1998). Bei beiden ist jedoch zu beachten, dass die zeitliche Distanz zwischen Wahrnehmung und Befragung die Messergebnisse stark beeinflusst (Kloss 2003, S. 162).

- Insbesondere angesichts der weiten Verbreitung von Recall-Messungen ist es bemerkenswert, dass bis heute darüber diskutiert wird, ob sie valide und reliable Messungen liefern. Einigkeit besteht weitgehend darüber, dass Recall-Werte nur wenig aussagekräftig sind, wenn nicht gleichzeitig qualitative Aspekte berücksichtigt werden (Pepels 2001, S. 176). Angesichts der Diskussion um die Zuverlässigkeit und Aussagekraft der Recall-Werte existieren zudem Überlegungen, ob Recognition dem Recall als Kenngröße vorzuziehen ist. Da jedoch unterschiedliche Aspekte mit den beiden Verfahren gemessen werden und es 
sowohl Personen mit einem stärkeren Recall-Gedächtnis als auch Personen mit einem stärkeren Recognition-Gedächtnis gibt (Hossinger 1982, S. 71), werden weiterhin beide Verfahren eingesetzt.

- Die Hauptkritik an Recognition-Tests richtet sich darauf, dass die Korrektheit der Antworten nicht überprüft werden kann und es in diesem Zusammenhang zu stark überhöhten Werten kommt, weil Menschen im Zweifel eher zustimmend antworten. Dem kann durch den Einsatz fiktiver bzw. unechter Werbemitteln (Erichson und Maretzki 1993, S. 548) bzw. von „Phantommarken“ begegnet werden.

- Zudem können Recall-Werte mit den Ergebnissen apparativer Testverfahren kombiniert werden, wie das folgende Beispiel zeigt: Erzielt eine Anzeige einen Markenrecall von 40 Prozent, dann kann dies zunächst als normales Ergebnis interpretiert werden. Zeigt die Blickregistrierung jedoch gleichzeitig, dass die zentralen Markeninformationen von nur 60 Prozent der Leser beachtet wurde, weist dies darauf hin, dass die Gestaltung noch Optimierungspotenzial aufweist - der Beitrag der Anzeige zur Markenaktualisierung kann noch gesteigert werden (von Keitz 1997).

Für die Markenführung ist die Markenbekanntheit (ausführlich Reinecke und Janz 2007, S. 254 f.) von besonderer Bedeutung, da sie als Anker von Assoziationen dient, Voraussetzung für das Entstehen von Vertrautheit und Zuneigung sowie ein Zeichen von Solidität und Engagement ist. Ferner bestimmt sie, ob die Marke im Awareness Set des Konsumenten ist bzw. ob sie bei der Kaufentscheidung in Betracht gezogen wird (Aaker 1992, S. 85). Grundsätzlich werden aktive und passive Markenbekanntheit unterschieden: Mittels Marken-Recall-Messung wird die aktive und mittels Marken-Recognition-Tests die passive Markenbekanntheit erhoben (Kroeber-Riel und Gröppel-Klein 2013, S. 455). Weitere Bekanntheitsarten (unbekannt, passiv, aktiv, top of mind, exklusiv) hat Aaker (1992, S. 83 f.) in einer Markenbekanntheitspyramide zusammengefasst.

\subsection{Einstellungen, Choice-Sets, Kaufabsicht und Likes/Dislikes zur Prognose von Konsumentenverhalten}

Für die Prognose des Konsumentenverhaltens (z. B. Kauf, Weiterempfehlung) sind Einstellungen zentrale Messgrößen, da sie wesentliche Verhaltensdeterminanten sind: Von ihnen hängt $\mathrm{ab}$, welche Informationen in die einzelnen Speicher gelangen und welche Wirkungen diese Informationen dort haben. Einstellungen werden als zeitlich relativ stabile Bereitschaften, auf ein bestimmtes Objekt wertend zu reagieren, verstanden und sind dementsprechend nur langfristig zu verändern (u. a. Kroeber-Riel und Gröppel-Klein 2013, S. 55 ff.). Ihre Rolle ist tendenziell so zu verstehen, dass eine Leistung, gegenüber der eine positive Einstellung besteht bzw. die grundsätzlich positiv bewertet wird, eher gekauft wird, als eine Leistung, die grundsätzlich eher negativ bewertet wird. Die Annahme, dass diese Richtung der Kausalität immer gegeben ist, kann jedoch nicht mehr aufrechterhalten werden: Teilweise bedingen 
auch Veränderungen im Kaufverhalten Einstellungsänderungen; bei Low Involvement-Produkten scheinen bei Erstkäufen Einstellungs- den Verhaltensänderungen, bei High Involvement-Produkten scheinen Verhaltens- den Einstellungsänderungen zu folgen (u. a. Kroeber-Riel und Gröppel-Klein, 2013, S. 247 ff.).

Die Präferenzbildung für ein bestimmtes Produkt erfolgt in mehreren Stufen (Awareness Set, Evoked Set etc.) und orientiert sich vor allem an Preis- und Qualitätsmerkmalen, so dass es für das Marketingmanagement wesentlich ist, entsprechende Preisschwellen und bestimmte Mindestanforderungen zu kennen (ausführlich Spiggle und Sewall 1987, S. 99 f.; Kroeber-Riel und Gröppel-Klein 2013, S. 339 f., S. 472 ff. und Reinecke und Janz 2007, S. 252). Für Analysen dieser Choice Sets eignen sich Instrumente, die Bekanntheits- und Imagemessungen mittels Befragung und Beobachtung kombinieren (ausführlich Spiggle und Sewall 1987, S. 100 ff.).

Darüber hinaus wird seit einigen Jahren in der Konsumentenforschung ein Konstrukt diskutiert und verstärkt eingesetzt, das dem tatsächlichen Verhalten näher ist, so dass ihm eine steigende Bedeutung in der Werbewirkungsforschung zukommt (u. a. Kroeber-Riel und Gröppel-Klein, 2013, S. 56 f., S. 248 ff.): Die Kaufintention, die neben den Einstellungen auch die subjektive Einschätzung der antizipierten Kaufsituation wie die Einschätzung des zum Kaufzeitpunkt für den Konsum verfügbaren Einkommens (,disposable income“), des voraussichtlichen Konsums oder die Wahl des Händlers umfasst. Zum Beispiel kann aus der Einstellung „Ich schätze das Produkt X sehr" die Kaufabsicht „Ich beabsichtige, das Produkt X für den Preis P noch in diesem Jahr beim Händler H zu kaufen“" werden. Die Einstellung als eine auf einen bestimmten Gegenstand bezogene Haltung wird somit für eine ganz bestimmte Handlungssituation differenziert und präzisiert. Die befragte Person wird gebeten, anzugeben, wie hoch die Wahrscheinlichkeit ist, dass sie ein bestimmtes Produkt innerhalb eines definierten Zeitraums erwirbt (u. a. Schweiger und Schrattenecker 2013, S. 391 f.). In der Regel soll sich die Person entscheiden, welches Element aus einem vorgegebenen Set von Alternativen sie wählen würde. Wird diese Messung nach Darbietung eines Kommunikationsmittels wiederholt, kann ermittelt werden, wie sich dieses auf die Kaufabsicht auswirkt (Nieschlag et al. 2002, S. 1115). Verschiedene Marktforschungsinstitute haben Tests (z. B. o. V. 1997, S. 20 f.) entwickelt, um die Kaufabsicht abzufragen.

Ferner wird die Reaktion auf die Werbung als Kenngröße der Werbewirkungskontrolle in Form von Likes und Dislikes und weiteren diagnostischen Fragen erhoben (Haley und Baldinger 2000, S. 116 ff.). Diese Größen spielen zum einen für die Vermeidung von Reaktanz und damit die Vermeidung des Abbruchs der Kommunikationsverarbeitung durch die Zielpersonen eine große Rolle. Zum anderen ist die Einstellung gegenüber der Werbung eine wichtige Determinante der Einstellung gegenüber der beworbenen Marke und der Kaufabsicht. Im Hintergrund steht hierbei das „Attitude toward the ad“-Modell (Lutz et al. 1983; weiterentwickelt durch MacKenzie und Lutz 1989). Demnach ist die Einstellung zu einer Werbemaßnahme eine zentrale vermittelnde Variable zwischen den direkten Auswirkungen der Werbemaßnahme und der Einstellung zu der beworbenen Marke sowie der Kaufabsicht. 


\subsection{Informations-, Beeinflussungs-, Kauf-, Wiederkauf- und Verwendungsverhalten zur Evaluation des Konsumentenverhaltens}

Für die Evaluation des Verhaltens von Zielpersonen in Form von Informations-, Beeinflussungs-, Kauf-, Wiederkauf- und Verwendungsverhalten kommen grundsätzlich mehrere Möglichkeiten wie Befragungen, Experimente oder Beobachtungen in Frage (hier und im Folgenden auch Steffenhagen 2008, S. 164 ff.).

Beobachtungen sind insbesondere bei Nutzungs- und Orientierungsverhalten relevant, wobei zwischen der Beobachtung von Verhaltensabläufen (z. B. Beobachtung der Reaktion von Kunden auf Werbung am Point of Sale/in Einzelhandelsgeschäften; auch videogestützt) und der Beobachtung von Verhaltensergebnissen (z. B. der Beobachtung von Abverkaufszahlen anhand von Scannerkassendaten) unterschieden werden kann. Ergänzend können die Konsumenten zu ihren Verhaltensabsichten (vor Betreten der Einkaufsstätte) und zu ihren Erlebnissen vor Ort befragt werden.

Informationsverhalten im Sinne von „Informationen einholen“ umfasst zum Beispiel das Anfordern von Prospekten und Preislisten, Messebesuche bzw. Besuche des Messestands, das Aufsuchen einer Einkaufsstätte, das Wahrnehmen eines persönlichen Beratungsgesprächs oder eine Probefahrt. Die Zurechnung solcher Wirkungen zur Kommunikation kann dadurch erleichtert werden, dass gewisse Response-Elemente verwendet werden (z. B. Coupons, Bestellkarten, Hotline, Social Media und ferner Gewinnspiele). Der Kontrolle dienen insbesondere die Registrierung schriftlich eingehender Anfragen (Antwortkartenrücklauf, Onlinerückmeldungen), die Zählung persönlicher Informanten (z. B. Standbesucherzählung bei Messen oder Ausstellungen) oder die Erfassung der Abrufhäufigkeit elektronisch verfügbarer Informationen.

Wirkungen der Kommunikation auf das Verwendungs- und Beeinflussungsverhalten zum Beispiel in Form von Erhöhung der verwendeten Menge oder Änderung/ Ausweitung der Verwendungssituationen (z. B. Verwendung eines längeren Zahnpastastreifens und Zähneputzen im Büro) lassen sich nur mittels speziell angelegter Befragungen bzw. Beobachtungen erheben, wobei die Weiterempfehlungsabsicht häufig auch in Kundenzufriedenheitsbefragungen erhoben wird.

Weitere Ziele der Marketingkommunikation können Größen sein wie Kundenbindung, „Share of Wallet“, Neukundengewinnung, Wiederkäufergewinnung oder Reduktion der Stornoquoten. Wie deutlich geworden ist, beruhen diese in der Regel nicht auf ,direkten“ Wirkungen der Kommunikation; sie sind damit keine direkten Zielgrößen der Kommunikation. Vielmehr müssen diese mittels geeigneter, zuvor aufgezeigter Indikatoren operationalisiert werden, um umgesetzt und kontrolliert werden zu können.

\subsection{Absatz als zentrale Größe zur Messung des Markterfolgs}

Kauf und Wiederkauf als zentrale Zielgrößen sind u. a. an den Absatzzahlen ablesbar, so dass es bei diesen Zielgrößen eine Überschneidung mit der letzten Wirkungsstufe gibt: mit der Markterfolgskonsequenz für den Anbieter, die meist mittels des 
realisierten Absatzes und/oder Umsatzes gemessen wird. Darüber hinaus sind folgende Kenngrößen üblich: Produktklassenwahl, Markenwahl, Lieferanten- bzw. Einkaufstättenwahl, Kaufmenge je Kauf, Kaufhäufigkeit, Kaufzeitpunkt, Käuferreichweite, Marktpenetration, Bedarfsdeckungsrate und Wiederkäuferpenetration (Steffenhagen 2008, S. 64 ff.).

Das Top-Management erwartet häufig einen Beitrag von Kommunikationsmaßnahmen zu Unternehmenszielen (v. a. Risikominimierung/Unabhängigkeit, Gewinn bzw. Profitabilität [langfristig, kurzfristig] und Wachstum [Umsatz, Absatz, Marktanteil]). Der Nachweis des Beitrags der Kommunikation zur Erreichung von Absatzund Umsatzzielen ist aufgrund zahlreicher Störfaktoren jedoch anspruchsvoll: Neben Störgrößen wie Auswirkungen von Konkurrenz- oder Preis-Promotionsmaßnahmen wird bereits die Messung der Kommunikationswirkungen durch Interdependenzeffekte (die gemessene Veränderung kann auf andere Maßnahmen zurückzuführen sein), Ausstrahlungseffekte (ausstrahlen auf andere Maßnahmen) und Carry-over-Effekte (zeitliche Abgrenzbarkeit der Kommunikationswirkungen ist problematisch) erschwert. Nicht selten bleiben deshalb die erforderlichen UrsacheWirkungszusammenhänge unklar.

Die Situation hat sich zumindest im Konsumgüterbereich jedoch mit Verbreitung der Scannerkassen seit den 1980ern deutlich verbessert. Auch konnte zumindest für die Konsumgüterbranche nachgewiesen werden, dass effektive Werbung unmittelbare (bzw. in einem Zeitraum von sieben Tagen bis sechs Monaten auftretende) Absatzeffekte hervorruft und dass kurzfristige Effekte unbedingt erforderlich sind, um einen langfristigen Werbeerfolg nachweisen zu können (Abraham und Lodish 1990, Lodish et al. 1995, S. G137 ff.; Blair und Schroiff 2001, S. 52 ff.). Rutschmann (2013) kritisiert allerdings, dass die meiste Massenkommunikation nicht zuletzt aufgrund der heutigen enormen Informationsflut kaum noch kaufverhaltenswirksame Effekte bewirkt.

Daher sollte stets versucht werden, über langfristige Messungen unternehmensund situationsspezifische Ursache-Wirkungszusammenhänge zu identifizieren, um Prognosen zu ermöglichen.

Geeignete Prognosemethoden basieren auf (Schweiger und Schrattenecker 2013, S. 393)

- den geäußerten Kaufabsichten der Konsumenten,

- dem unter Testbedingungen (Experimentalbedingungen) beobachteten Kaufverhalten und/oder

- Absatzzahlen der Vergangenheit (v. a. Panelerhebungen, Scannerkassendaten und Auftragseingangsstatistiken; die Auswertung erfolgt v. a. mittels Marktreaktionsfunktionen/ökonometrischen Modellen).

\subsubsection{Testverfahren zur Ermittlung eines Kausalzusammenhangs zwischen Kommunikation und Umsatz}

Mit Hilfe experimenteller Untersuchungen kann versucht werden, einen Kausalzusammenhang zwischen Input (Kommunikation) und Output (z. B. Umsatz) nachzuweisen. 
In der Praxis werden diese aus Gründen der Kosten- und Komplexitätsreduktion teilweise derart durchgeführt, dass in einigen Verkaufsgebieten höhere Aufwendungen und in anderen Gebieten weniger Aufwendungen vorgenommen und anschließend die Ergebnisse verglichen werden (Kotler et al. 2007, S. 794 ff.). Auch wird die Werbekampagne nicht selten zunächst in einzelnen Städten oder Regionen durchgeführt, bevor sie national mit großem Budget anläuft; bei internationalen Kampagnen werden häufig kleinere Länder als Testmärkte gewählt.

Durch Einsatz systematischer Tests sollten jedoch zuverlässigere Ergebnisse erzielt werden können. Marketingrelevante Testverfahren sind Produkttests, Storetests, Testmärkte und die für den Kommunikationsbereich (insbesondere bei schnelldrehenden Konsumgütern) zentralen Testmarktersatzverfahren. Während Produkttests vor allem auf den Ge- und Verbrauch von Produkten gerichtet sind, werden im Rahmen von Storetests auch kommunikative Point-of-Sale-Aspekte untersucht (v. a. Verpackungselemente oder Promotionmaßnahmen). Bei der Überprüfung der Marktchancen von Produkten weisen Ergebnisse aus Testmärkten die größte Realitätsnähe auf, weil das neue Produkt in einem realen, jedoch regional abgegrenzten Teilmarkt eingeführt und sämtliche für die Markteinführung vorgesehenen Maßnahmen (einschließlich Testwerbung) eingesetzt werden (Koch 2012, S. 102 ff.). Aufgrund einiger Nachteile von Testmärkten werden in der Praxis jedoch vor allem Testmarktersatzverfahren eingesetzt (Mini-Testmärkte und Testmarkt-Simulationen; Koch 2012, S. 109 ff.).

Instrumente wie der BehaviorScan der GfK erlauben das parallele Auswerten der Daten aus Mini-Testmärkten (Scannerkassendaten usw.) und Daten der elektronischen Fernsehpanels mittels Television-Meter (in Deutschland dem GfK-Meter). Im Grunde ist der BehaviorScan ein um die Werbewirkungskontrolle erweiterter MiniTestmarkt. Auf diese Weise können unter anderem kommunikationsrelevante Fragen beantwortet werden: Wer kauft das neue Produkt? Wie wirkt TV-Werbung auf den Abverkauf? Welches Media-Spending-Level ist optimal? Welche TV-Werbung steigert den Abverkauf am stärksten? Ferner kann Folgendes bestimmt werden: Durchsetzungsfähigkeit gegenüber anderen Spots, Überzeugungskraft in der Zielgruppe, Kaufbereitschaft des Zielpublikums und Funktionieren des Botschaftstransports, weil Klarheit, Verständnis, Prägnanz und Produktpositionierung optimiert sind oder nicht. Die BehaviorScan-Haushaltspanels sind repräsentativ für Deutschland und Frankreich. Die 3500 teilnehmenden Haushalte in Deutschland erhalten eine GfK-Identifikationskarte, die sie beim Bezahlen in den teilnehmenden Supermärkten vorzeigen. Auf diese Weise kann das Kaufverhalten präzise erhoben werden. Gleichzeitig kann gesteuert und kontrolliert werden, welche Werbung die teilnehmenden Personen im Fernsehen sehen: Mit Hilfe der Targetable TV-Technologie ist es möglich, bei einem Teil der Panelhaushalte die nationalen TV-Spots mit Testwerbung zu überstrahlen und einem anderen Teil der Panelhaushalte (der Kontrollgruppe) weiterhin die aktuelle Werbung zu zeigen, so dass neue Werbungen getestet werden können (Koch 2012, S. 110 ff.). In Deutschland werden neben dem GfK-BehaviorScan (in Haßloch) die Mini-Testmärkte GfK-Erim-Panel (nahe Berlin, Hannover, Köln und Nürnberg) und das Telerim Panel von ACNielsen angeboten (in Buxtehude und Bad Kreuznach; u. a. Bere-koven et al. 2009, S. 160 ff.). 


\subsubsection{Marktreaktionsfunktionen und Werberesponsemodelle zur Effektivitäts- und Effizienzbewertung einzelner Marketinginstrumente}

Zur Effektivitäts- und Effizienzbewertung einzelner Marketinginstrumente haben sich ferner Marktreaktionsfunktionen durchgesetzt. Diese bilden das Kaufverhalten der Konsumenten bei unterschiedlichen Angebotsbedingungen ab, indem sie einen Zusammenhang zwischen Input- und Outputgrößen eines Marktes herstellen (Balderjahn 1993, S. 30).

Im Marketing bilden sie den Zusammenhang zwischen den Inputgrößen „Einsatz von Marketinginstrumenten“ und „Marketingaktivitäten der Konkurrenten“ auf der einen Seite und abhängigen Größen bzw. Outputgrößen wie Marktanteil oder Absatzvolumen unter Berücksichtigung gesamtwirtschaftlicher und situativer Faktoren auf der anderen Seite ab, so dass Prognosen bezüglich der Outputgrößen getroffen werden können (Balderjahn 1993, S. 30). Mittels Marktreaktionsfunktionen sollen möglichst valide Prognosen über den Erfolg einzelner Marketinginstrumente getroffen werden, um die Entscheidungs- und Planungsqualität zu erhöhen (auch Balderjahn 1993, S. 31). Welcher Funktionstyp unterstellt wird, hängt davon ab, welche Theorien und somit welche Modelle berücksichtigt werden (siehe Werberesponsefunktionen).

Für die Berechnung der Marktreaktionsfunktionen werden multivariate Verfahren wie die Regressionsanalyse eingesetzt, so dass vereinzelt (z. B. Erichson und Maretzki 1993, S. 537) auch von einem ökonometrischen Ansatz gesprochen wird, wenn Marktreaktionsfunktionen im Marketing gemeint sind. Zu beachten ist hierbei, dass die Regressionsanalyse zwar ein häufig zur Berechnung der Marktreaktionsfunktionen vorgeschlagenes Verfahren ist, diese nach Steffenhagen (1978, S. 18) jedoch unzureichend ist. Sie reflektiert meist keine Erklärungsmodelle, sondern nur Strukturen empirischer Regelmäßigkeit, da sie auf Stimulus-response-Modellannahmen (SR-Modelle) basiert. Verfahren, die den Stimulus-Object-Response-Modellannahmen gerecht werden, sind dagegen die Conjoint-Analyse und die diskrete Entscheidungsanalyse (Balderjahn 1993, S. 32).

Ein Beispiel für eine sehr einfache Marktreaktionsfunktion ist die Preis-AbsatzFunktion bzw. Preisresponsefunktion. Werbesresponsefunktionen sind wesentlich komplexer, weil sie weitere Inputgrößen und Rahmenbedingungen berücksichtigen müssen: Erstens sind alle auf den Markt gerichteten Maßnahmen eines Unternehmens und der Konkurrenz zu berücksichtigen. Zweitens muss die Funktion dynamisch formuliert werden, da teilweise Carry-Over-Effekte zu berücksichtigen sind, so dass idealerweise Zeitreihendaten zur Verfügung stehen sollten. Drittens muss die Funktion stochastisch bzw. eine Wahrscheinlichkeitsfunktion sein, da nicht alle Wirkungsgrößen berücksichtigt werden können und die unerklärten Größen als zufällige Störvariablen erfasst werden müssen (Hansmann und Diller 2001, S. 1066 f.). Zu berücksichtigen sind dabei zahlreiche Aspekte wie saisonale oder lebenszyklusbedingte Schwankungen der Kommunikationswirkungen.

Im Werbebereich werden zu diesem Zweck Werberesponsefunktionen eingesetzt. Relevante Inputgrößen umfassen sowohl Größe und Schaltungsfrequenz als auch die Höhe des eingesetzten Werbebudgets. Outputgrößen umfassen unternehmenserfolgsrelevante Größen wie Umsatz und Absatz sowie nichtmonetäre Werbeziele 
wie Recall, Einstellungen oder Kaufabsicht (Simon und Arndt 1980, S. 12; Stewart 1989, Evaluation der Werberesponsefunktionen; Steffenhagen 2000, S. 195 f., Arten der Werberesponsefunktion).

Grundsätzlich werden in der Werbewirkungsforschung vier verschiedene Funktionstypen unterschieden: lineare, exponentielle (konvexer Kurvenverlauf), degressive (konkaver Kurvenverlauf) und logistische (S-förmiger Kurvenverlauf), wobei vor allem die degressive und die S-förmige eine wichtige Rolle spielen (Nieschlag et al. 2002, S. 1105 ff.; Bruhn 2013 S. 58 ff.). Welche Funktion unterstellt wird, hängt von den jeweils zugrunde gelegten theoretischen Annahmen ab. Häufig wird davon ausgegangen, dass sich das Verhältnis von Kommunikationsbudget und Absatzentwicklung am besten durch einen S-förmigen Verlauf abbilden lässt, da dieser die folgenden, zentralen Annahmen berücksichtigt (Simon und Arndt 1980, S. 12 f.): Erstens, dass ein Mindestwerbedruck und damit ein Mindestbudget erforderlich ist. Zweitens, dass im Werbebereich in der Regel von einem abnehmenden Grenzertrag bei zunehmender Kommunikationsintensität bzw. Sättigungstendenzen und einer nur begrenzt steigerbaren Kaufbereitschaft, ausgegangen werden sollte (Nieschlag et al. 2002, S. 1106 f.). Gelegentlich wird auch ein degressiver (konkaver) Kurvenverlauf unterstellt, der ebenfalls den abnehmenden Grenzertrag berücksichtigt (Simon und Arndt 1980, S. 12 f.). Bisher konnten jedoch noch keine verallgemeinerbaren Funktionsverläufe bestimmt werden, da diese stets stark von der jeweiligen Situation des Unternehmens und des Marktes abhängen. Ein häufig genannter Grund sind die viel zitierten Störgrößen, die das Ausweisen eines Kausalzusammenhangs erschweren.

Werberesponsemodelle für die Evaluation der Wirkung der Budgethöhe auf den Markterfolg (z. B. Rao und Miller 1975 und Little 1979) wurden seit den 1950ern/ 1960ern entwickelt und häufig für die Werbebudgetierung empfohlen, wobei sich drei Gruppen identifizieren lassen: Zum einen a priori Modelle, die weniger auf Daten basieren, sondern eher die Intuition berücksichtigen, um eine generelle Wirkungsstruktur aufzuzeigen. Zu diesem Ansatz gehören die Modelle von Vidale und Wolfe (1957), von Nerlove und Arrow (1962) sowie von Little (1966, 1975). Zum anderen die statistische oder ökonometrische Tradition mit Modellen, die in der Regel mit einer spezifischen Datenbasis wie Zeitreihen von Absatz oder Marktanteil und Werbung arbeiten. Zu diesen gehören beispielsweise die Modelle von Bass und Clarke (1972), Montgomery und Silk (1972) sowie Lambin (1976). Ferner liegen Mischmodelle vor, die von weiterentwickelten a priori Modellen ausgehen und versuchen, diese mittels statistischer Methoden zu bestätigen und zu evaluieren (Little 1979, S. 644 ff., Wang et al. 2013, 22 ff.).

\section{$4 \quad$ Fazit: Wirksamkeit und Wirtschaftlichkeit der Kommunikation durch Controlling sicherstellen}

Ein Erfolgsnachweis der Marketingkommunikation ist wie aufgezeigt nicht nur notwendig, sondern auch möglich: Es existieren inzwischen zahlreiche Methoden, Instrumente und Kennzahlen, die dabei helfen, die Wirksamkeit und die Wirtschaftlichkeit 
der Kommunikation sicherzustellen und auszuweisen. Entscheidend ist allerdings, dass eine differenzierte, instrumenten- bzw. aufgabenbezogene Planung der Kommunikationsziele erfolgt, denn ohne solche verbindliche Zielplanung ist weder eine (klassische) Kontrolle noch ein (umfassenderes) Controlling der Marketingkommunikation möglich.

\section{Literatur}

Aaker, D.A. 1992. Management des Markenwerts. Frankfurt a. M. /New York. Übers. Friedrich Mader. 1991. Managing Brand Equity. New York: Campus Verlag.

Abraham, M. M., und L. M. Lodish. 1990. Getting the most out of your advertising and promotion. Harvard Business Review 68(3): 50-60.

Ambler, T. 2000. Persuasion, pride and prejudice: How ads work. International Journal of Advertising 19(3): 299-315.

Backhaus, K., und M. Voeth. 2009. Industriegütermarketing, 9. Aufl. München: Vahlen.

Balderjahn, I. 1993. Marktreaktionen von Konsumenten: ein theoretisch-methodisches Konzept zur Analyse der Wirkung marketingpolitischer Instrumente. Berlin: Duckner \& Humblot.

Bass, F.M., und D.G. Clarke. 1972. Testing distributed lag models of advertising effect. Journal of Marketing Research 9(3): 298-308.

Bauer, H. H., U. Meeder, und J. Jordan. 2000a. Ausgewählte Instrumente des Werbecontrolling, Reihe Management Know-How, Nr. M 59, Universität Mannheim, Institut für Marktorientierte Unternehmensführung. Mannheim.

Bauer, H. H., U. Meeder, und J. Jordan. 2000b. Eine Konzeption des Werbecontrolling, Reihe Wissenschaftliche Arbeitspapiere, Nr. W 46, Universität Mannheim, Institut für Marktorientierte Unternehmensführung. Mannheim.

Behrens, G. 1996. Werbung: Entscheidung - Erklärung - Gestaltung. München: Vahlen.

Berekoven, L., W. Eckert, und P. Ellenrieder. 2009. Marktforschung. Methodische Grundlagen und praktische Anwendung, 12. Aufl. Wiesbaden: Gabler.

Bergkvist, L. 2000. Advertising effectiveness measurement: Intermediate constructs and measures. Stockholm: Stockholm School of Economics.

Blair, M. H., und H.-W. Schroiff. 2001. Wann wirkt Werbung - heute, morgen oder gar nicht? Planung \& Analyse 1:52-57.

Böcker, F. 1988. Marketing-Kontrolle. Stuttgart et al: Kohlhammer.

Brettschneider, F., B. Ostermann, und U. Seidel. 2007. Erfolgsfaktoren für KommunikationsControlling. Wie Kommunikationsmanager und Controller mit Intangible Performance Management gemeinsam Neuland erschließen können. Controller Magazin, Bd. 32.

Bruhn, M. 2013. Kommunikationspolitik - Systematischer Einsatz der Kommunikation für Unternehmen, 7. Aufl. München: Vahlen.

Cramphorn, S. 2004. What advertising testing might have been, if we had only known. Journal of Advertising Research 44(2): 170-180.

Day, G., und L. Fahey. 1988. Valuing market strategies. Journal of Marketing 52(3): 45.

Doyle, P. 2000. Value-based marketing. Journal of Strategic Marketing 8(4): 299-311.

Ellinghaus, U. 2000. Werbewirkung und Markterfolg - Marktübergreifende Werbewirkungsanalysen. München: Oldenbourg.

Erichson, B., und J. Maretzki. 1993. Werbeerfolgskontrolle, Hrsg. R. Berndt und A. Hermanns, 521-560. Gabler.

Farris, P. W., N. T. Bendle, P. E. Pfeifer, und D. J. Reibstein. 2010. Marketing metrics: The definitive guide to measuring marketing performance. 2. Aufl. Upper Saddle River: FT Press. 
Fenwick, I., und M. D. Rice. 1991. Reliability of continuous measurement copy-testing methods. Journal of Advertising Research 31(1): 23-29.

Haley, R., und A. L. Baldinger. 2000. The ARF copy research validity project. Journal of Advertsing Research 41(6): 114-135.

Hansmann, K.-W., und H. Diller. 2001. Marktreaktionsfunktionen. In Vahlens grosses Marketinglexikon, Hrsg. H. Diller, 2. Aufl., 1066-1067. München: Vahlen.

Hermanns, A., und C. Marwitz. 2008. Sponsoring: Grundlagen, Wirkungen, Management, Markenführung, 3. Aufl. München: Vahlen.

Horváth, P. 2012. Controlling, 12. Aufl. München: Handelsblatt Fachmedien.

Hossinger, H.-P. 1982. Pretests in der Marktforschung. Die Validität von Pretestverfahren der Markforschung unter besonderer Berücksichtigung der Tachistoskopie. Würzburg: PhysicaVerlag.

Huhn, J., und J. Sass. 2011. Positionspapier: Kommunikations-Controlling. Bonn.

Janz, S. 2009. Performance Management am Beispiel der Marketingkommunikation: KonzeptionErfolgsauswirkungen - Determinanten. St. Gallen: Südwestdeutscher Verlag für Hochschulschriften.

Kloss, I. 2003. Werbecontrolling - Konzepte, Instrumente, Fallbeispiele. Gernsbach: Deutscher Betriebswirte-Verlag.

Koch, J. 2012. Marktforschung: Grundlagen und praktische Anwendungen, 6. Aufl. München: Oldenbourg.

Köhler, R. 2006. Marketing-Controlling: Konzepte und Methoden. In Handbuch Marketingcontrolling, Hrsg. S. Reinecke und T. Tomczak, 2. Aufl., 39-61. Wiesbaden: Gabler.

Kotler, P., und K. L. Keller. 2012. Marketingmanagement, 14. Aufl. Essex: Pearson.

Kotler, P., K. L. Keller, und F. Bliemel. 2007. Marketing-Management. Strategien für wertschaffendes Handeln, 12. Aufl. Stuttgart: Pearson.

Krishnan, H. S., und D. Chakravarti. 1999. Memory measures for pretesting advertisements: An integrative conceptual framework and a diagnostic template. Journal of Consumer Psychology 8 (1): $1-37$.

Kroeber-Riel, W., und A. Gröppel-Klein. 2013. Konsumentenverhalten, 10. Aufl. München: Vahlen.

Kroeber-Riel, W., und F.-R. Esch. 2011. Strategie und Technik der Werbung, Verhaltenswissenschaftliche und neurowissenschaftliche Ansätze, 7. Aufl. Stuttgart: Kohlhammer.

Kuß, A., und T. Tomczak. 2007. Käuferverhalten, 4. Aufl. Stuttgart: UTB.

Lambin, J.-J. 1976. Advertising, competition and market conduct in oligopoly over time. Amsterdam: North Holland.

Lasslop, I. 2003. Effektivität und Effizienz von Marketing-Events: Wirkungstheoretische Analyse und empirische Befunde. Wiesbaden: Gabler.

Little, J. D. C. 1966. A model of adaptive control of promotional spending. Operations Research 14 (6): 1075-1097.

Little, J. D. C. 1975. Brandaid: A marketing-mix model, parts 1 and 2. Operations Research 23(4): 628-673.

Little, J. D. C. 1979. Aggregate advertising models: The state of the art. Operations Research 27(4): 629-667.

Lodish, L. M., M. M. Abraham, J. Livelsberger, B. Lubetkin, B. Richardson, und M.E. Steven. 1995. A summary of fifty-five in-market experimental estimates of the long-term effect of TV advertising. Marketing Science 14(3): G133-G140.

Lutz, R., S. MacKenzie, und G. E. Belch. 1983. Attitute toward the ad as a mediator of advertising effectiveness: Determinants and consequences. Advances in Consumer Research 10(1): 532-539.

MacKenzie, S., und R. Lutz. 1989. An empirical examination of the structural antecedents of attitude toward the ad in an advertising pretesting context. Journal of Marketing 53: $48-65$. 
Montgomery, D. B., und A. J. Silk. 1972. Estimating dynamic effects of marketing communications expenditures. Management Science 18(10): 485-501.

Neff, J. 2005. 200 P\&G ads entered for Cannes. But frustrated agency creatives don't expect big wins, AdAge online edition. Zugegriffen am 13.06.2005 http://www.adadge.com (Artikel-ID: AAQ64L).

Nerlove, M., und K. J. Arrow. 1962. Optimal advertising policy under dynamic conditions. Economica 29: 129-142.

Neumann, P. 2013a. Handbuch der Markt- und Werbepsychologie: Grundlagen, Wahrnehmung, Lernen, Aktivierung, Image-Positionierung, Verhaltensbeeinflussung, Kreativität. Bern: Huber.

Neumann, P. 2013b. Handbuch der psychologischen Marktforschung. Bern: Huber.

Nickel, O. 2001. Zur Diskussion des Recalls als Indikator für den Werbeerfolg. Planung \& Analyse, Hrsg. Ralf Meyer de Groot, Tanja Pallek und Elmar Haimer, 13(5): $70 \mathrm{ff}$.

Nieschlag, R., E. Dichtl, und H. Hörschgen. 2002. Marketing, 19. Aufl. Berlin: Duncker \& Humblot Berlin.

O. V. 1997. Werbetrackinginstrumente. Eine Analyse der Standardinstrumente zur Werbeerfolgskontrolle. Berlin.

O'Sullivan, D., und A. V. Abela. 2007. Marketing performance measurement ability and firm performance. Journal of Marketing 71(2): 79-93.

Pepels, W. 2001. Kommunikations-Management. Marketing-Kommunikation vom Briefing bis zur Realisation, 4. Aufl. Stuttgart: Schäffer-Poeschel.

Rao, A. G., und P. B. Miller. 1975. Advertising/sales response functions. Journal of Advertising Research 15(2): 7-15.

Reinecke, S. 2004. Marketing Performance Management - Empirisches Fundament und Konzeption für ein integriertes Marketingkennzahlensystem. Wiesbaden: Deutscher Universitätsverlag.

Reinecke, S. 2014. Return on Marketing 2014: Sicherstellen der Marketing Performance in der Praxis. St. Gallen: Thexis.

Reinecke, S., und S. Janz. 2007. Marketingcontrolling. Sicherstellen von Marketingeffektivität und -effizienz. Stuttgart: Kohlhammer.

Rust, R. T., T. Ambler, G. S. Carpenter, V. Kumar, und R. K. Srivastava. 2004. Measuring marketing productivity: Current knowledge and future directions. Journal of Marketing 68(4): 76-89.

Rutschmann, M. 2013. Abschied vom Branding: Wie man Kunden wirklich ans Kaufen führt-mit Marketing, das sich an Kaufprozessen orientiert, 2. Aufl. Wiesbaden: Springer Gabler.

Schwaiger, M. (2006): Wirkungskontrolle kommunikationspolitischer Maßnahmen. In: Handbuch Marketingcontrolling, Hrsg. S. Reinecke und T. Tomczak, 2. Aufl., 521-548. Wiesbaden: Gabler.

Schweiger, G., und G. Schrattenecker. 2013. Werbung: Eine Einführung, 8. Aufl. Konstanz, München: UTB.

Siegwart, H., S. Reinecke, und S. Sander. 2010. Kennzahlen für die Unternehmensführung, 7. Aufl. Bern: Haupt Verlag.

Simon, J. L., und J. Arndt. 1980. The shape of the advertising response function. Journal of Advertising Research 20(4): 11-28.

Spiggle, S., und M. A. Sewall. 1987. A choice sets model of retail selection. Journal of Marketing 51(4): 97-111.

Stapel, J. 1998. Recall and recognition: A very close relationship. Journal of Advertising Research 38(4): 41-45.

Steffenhagen, H. 1978. Wirkungen absatzpolitischer Instrumente. Stuttgart: Schäffer-Poeschel Verlag.

Steffenhagen, H. 2000. Wirkungen der Werbung. Konzepte - Erklärungen - Befunde, 2. Aufl. Aachen: Mainz.

Steffenhagen, H. 2008. Marketing - Eine Einführung, 6. Aufl. Stuttgart: Kohlhammer. 
Stewart, D. W. 1989. Measures, methods, and models in advertising research. Journal of Advertising Research 29(3): 54-60.

Trommsdorff, V. 2003. Werbe-Pretests - Praxis und Erfolgsfaktoren. Hamburg: Gruner+Jahr.

Vidale, M. L., und H. B. Wolfe. 1957. An operations research study of sales response to advertising. Operations Research 5(3): 370-381.

von Keitz, B. 1997. Kommunikations-Tests mit apparativer Unterstützung - the State of the Art. Planung \& Analyse 2:40 ff.

Wang, M., Q. Gou, C. Wu, und L. Liang. 2013. An aggregate advertising response model based on consumer population dynamics. Journal of Applied Management Science 5(1): 22-38.

Weber, J., und U. Schäffer. 2008. Einführung in das Controlling, 12. Aufl. Stuttgart: SchäfferPoeschel Verlag. 